\title{
DECOMPOSITIONS OF BEURLING TYPE FOR $E_{0}$-SEMIGROUPS
}

\author{
ROLF GOHM \\ Department of Mathematics \\ University of Reading \\ Whiteknights, PO Box 220 \\ Berkshire, RG6 6AX, UK \\ E-mail:r.gohm@reading.ac.uk
}

\begin{abstract}
We define tensor product decompositions of $E_{0}$-semigroups with a structure analogous to a classical theorem of Beurling. Such decompositions can be characterized by adaptedness and exactness of unitary cocycles. For CCR-flows we show that such cocycles are convergent.
\end{abstract}

Introduction. A well-known theorem of Beurling characterizes invariant subspaces of the right shift on $\ell^{2}(\mathbb{N})$ by inner functions in the unit disc. In this case the restriction of the right shift to a nontrivial invariant subspace is automatically conjugate (unitarily equivalent) to the original shift. This interesting self-similar structure is fundamental in many respects. It is the prototype of a very fruitful interaction between operator theory and function theory, see for example $[\mathrm{Ni}, \mathrm{FF}]$.

In this paper we want to study a somewhat analogous self-similar structure for operators on a different level. While the original setting concerns isometries and decompositions of the Hilbert space into direct sums, we want to study $E_{0}$-semigroups, i.e., pointwise weak*-continuous semigroups of unital $*$-endomorphisms of $\mathcal{B}(\mathcal{H})$ for some complex separable Hilbert space $\mathcal{H}$ (cf. [Ar]), and decompositions of the Hilbert space into tensor products. To make the analogy visible, we present in Section 1 the Nagy-Foias functional model for $*$-stable contractions and their characteristic functions in a suitable way and in particular we emphasize a limit formula for the characteristic function which is not made explicit in the standard presentations. This analogy motivates the definition of decompositions of Beurling type for $E_{0}$-semigroups in Section 2. It is then shown that there is a reformulation in terms of unitary cocycles for amplifications of the $E_{0}$-semigroup. Relevant properties of the cocycles are adaptedness and exactness.

2000 Mathematics Subject Classification: Primary 46L55, 47D03; Secondary 81S25.

Key words and phrases: Beurling, $E_{0}$-semigroup, unitary cocycle, adapted, exact.

The paper is in final form and no version of it will be published elsewhere. 
In fact, if the $E_{0}$-semigroup is a CCR-flow (cf. [Ar]), then this leads to a setting which has been extensively studied by quantum probabilists (cf. [Pa]). We have a tensor product of an initial Hilbert space with a symmetric Fock space over $L^{2}[0, \infty)$, maybe with a multiplicity space $k$. Unitary cocycles of amplifications of the quantized shift can be used to construct quantum stochastic Markov processes in the form of socalled Evans-Hudson flows. In many cases the cocycles can be defined by quantum stochastic differential equations based on an integration theory initiated by Hudson and Parthasarathy. Pioneering work in establishing connections with $E_{0}$-semigroups has been done by Bhat in [Bh]. See also [Li] for an up-to-date introduction to the classification of various kinds of cocycles in this setting.

In this paper we do not adopt this differential point of view. In fact, it is an interesting task to relate our arguments to it and we shall do that elsewhere. The question posed by decompositions of Beurling type concerns conjugacy between the flow on the Fock space and the flow obtained by a cocycle perturbation of its amplification. It seems that this question has not been studied systematically. Here our analogy to the Nagy-Foiaş functional models turns out to be useful. In Section 3 we introduce the notion of a convergent unitary cocycle as a sufficient criterion for exactness. The main result of Section 4 is that for CCR-flows we have a converse, so that decompositions of Beurling type are characterized by the occurrence of convergent cocycles. The analogy to Section 1 becomes very explicit at this point and we have a kind of dictionary between these structures. In particular, the role of the $*$-stable contraction is now played by a semigroup of unital completely positive maps with an absorbing vector state.

Similar results for single completely positive maps and endomorphisms, i.e., with a discrete time parameter, have been obtained by the author in [Go], Chapter 2, where also some additional motivation for studying this kind of problems can be found. As may be expected, we can often use the insight from the discrete case but at some points we need to be more careful to choose the correct cocycles. For CCR-flows much is known about that and therefore our results are more complete in this case. The success story of Beurling's theorem indicates, in our view, that the study of decompositions of Beurling type may be helpful in the process of deciphering the structure of more general $E_{0}$-semigroups.

1. Characteristic functions as limits. Let $\mathcal{H}$ be a complex separable Hilbert space and $T: \mathcal{H} \rightarrow \mathcal{H}$ a contraction. We have defect operators $D=\sqrt{\mathbb{I}-T^{*} T}$ and $D_{*}=$ $\sqrt{\mathbb{I}-T T^{*}}$ and defect spaces $\mathcal{D}=\overline{D \mathcal{H}}$ and $\mathcal{D}_{*}=\overline{D_{*} \mathcal{H}}$. Then

$$
R=\left(\begin{array}{cc}
T & D_{*} \\
D & -T^{*}
\end{array}\right): \mathcal{H} \oplus \mathcal{D}_{*} \rightarrow \mathcal{H} \oplus \mathcal{D}
$$

is a unitary called the rotation matrix.

Using $H^{2}(\mathcal{D})$, the Hardy space of the unit disc with values in $\mathcal{D}$, we can write the minimal isometric dilation of $T$ as

$$
U: \mathcal{H} \oplus H^{2}(\mathcal{D}) \rightarrow \mathcal{H} \oplus H^{2}(\mathcal{D}), \quad h \oplus f \mapsto T h \oplus(D h+z f),
$$

where $z$ is the complex variable so that multiplication by $z$ represents a one-sided right shift. The following factorization of powers of $U$ is instructive. We denote by $H^{2}\left(\mathcal{D}_{*, k}\right)$ 
the space $H^{2}(\mathcal{D})$ with $\mathcal{D}$ in all levels up to $k$ replaced by $\mathcal{D}_{*}$, in other words, if $\sum_{n=0}^{\infty} a_{n} z^{n}$ is in $H^{2}\left(\mathcal{D}_{*, k}\right)$ then $a_{n} \in \mathcal{D}_{*}$ for $n \leq k$ and $a_{n} \in \mathcal{D}$ for $n>k$. For $k \in \mathbb{N}_{0}$ define

$$
\begin{gathered}
R_{k}: \mathcal{H} \oplus H^{2}\left(\mathcal{D}_{*, k}\right) \rightarrow \mathcal{H} \oplus H^{2}\left(\mathcal{D}_{*, k-1}\right), \\
h \oplus\left(\sum_{n=0}^{\infty} a_{n} z^{n}\right) \mapsto\left(T h+D_{*} a_{k}\right) \oplus\left(\sum_{n=0}^{k-1} a_{n} z^{n}+\left(D h-T^{*} a_{k}\right) z^{k}+\sum_{n=k+1}^{\infty} a_{n} z^{n}\right),
\end{gathered}
$$

i.e., $R_{k}$ is a kind of leg-numbering notation indicating $R$ acting on $\mathcal{H}$ and on the $k$-th level on the right. Then it is easy to check that for all $n \in \mathbb{N}$

$$
U^{n}(h \oplus f)=R_{0} \ldots R_{n-1}\left(h \oplus z^{n} f\right)
$$

(where we identify $\mathcal{H} \oplus 0$ in $\mathcal{H} \oplus \mathcal{D}_{*}$ and $\mathcal{H} \oplus \mathcal{D}$ )

$$
=T^{n} h \oplus\left(D T^{n-1} h+D T^{n-2} h z+\ldots+D h z^{n-1}+z^{n} f\right) .
$$

A contraction is called $*$-stable if $T^{* n} h \rightarrow 0$ if $n \rightarrow \infty$ for all $h \in \mathcal{H}$. Equivalent characterizations: (1) The minimal isometric dilation $U$ of $T$ is (unitarily equivalent to) a one-sided shift $S$. (2) $T^{*}$ is a restriction of $S^{*}$. To show these (obviously sufficient) conditions for a *-stable contraction $T$ one checks that

$$
C: \mathcal{H} \rightarrow H^{2}\left(\mathcal{D}_{*}\right), \quad h \mapsto D_{*}\left(\mathbb{I}-z T^{*}\right)^{-1} h,
$$

is an isometry which can be used to embed $\mathcal{H}$ into $H^{2}\left(\mathcal{D}_{*}\right)$. Because $S^{*} C=C T^{*}$, where $S$ is multiplication by $z$ in $H^{2}\left(\mathcal{D}_{*}\right)$, we have $(2)$. See [FF], Chapter IX, Theorem 6.4, where it is also shown how to extend $C$ to a unitary $W$ between the two dilation spaces intertwining $U$ and $S$, i.e., $W U=S W$. Explicitly,

$$
W: \mathcal{H} \oplus H^{2}(\mathcal{D}) \rightarrow H^{2}\left(\mathcal{D}_{*}\right), \quad h \oplus f \mapsto C h+\Theta_{T} f,
$$

where $\Theta_{T}(z)=-T+\left.z D_{*}\left(\mathbb{I}-z T^{*}\right)^{-1} D\right|_{\mathcal{D}}$ gives an inner function $\Theta_{T}$ with values in the bounded operators from $\mathcal{D}$ to $\mathcal{D}_{*}$. It is called the characteristic function of $T$. The $S$-invariant subspace $(C \mathcal{H})^{\perp}$ in $H^{2}\left(\mathcal{D}_{*}\right)$ can be written as $\Theta_{T} H^{2}(\mathcal{D})$, which corresponds to an operator version of Beurling's theorem. For $T$ we have a functional model on a $S$-coinvariant subspace. We want to call attention to the limit formula

$$
\hat{W}=\lim _{n \rightarrow \infty} R_{n-1}^{*} \ldots R_{0}^{*} .
$$

Note that $R_{n-1}^{*} \ldots R_{0}^{*}$ is nothing but the adjoint of the product occurring in the formula for $U^{n}$. Here we have a limit of unitaries and we assert that this limit is an isometry $\hat{W}$ onto $0 \oplus H^{2}\left(\mathcal{D}_{*}\right)$ which corresponds to the unitary $W: \mathcal{H} \oplus H^{2}(\mathcal{D}) \rightarrow H^{2}\left(\mathcal{D}_{*}\right)$ introduced above.

It is not difficult to check this limit formula by direct computation. In fact, by induction

$$
\begin{gathered}
R_{n-1}^{*} \ldots R_{0}^{*}\left(h \oplus \sum_{k=0}^{\infty} a_{k} z^{k}\right)=\left(T^{* n} h+T^{*(n-1)} D a_{0}+\ldots+D a_{n-1}\right) \\
\oplus \quad\left[\left(D_{*} h-T a_{0}\right)+\left(D_{*} T^{*} h+D_{*} D a_{0}-T a_{1}\right) z\right. \\
\left.+\ldots+\left(D_{*} T^{*(n-1)} h+D_{*} T^{*(n-2)} D a_{0}+\ldots-T a_{n-1}\right) z^{n-1}+\sum_{k=n}^{\infty} a_{k} z^{k}\right] .
\end{gathered}
$$


The first summand of the orthogonal sum converges to zero as $n \rightarrow \infty$ because $T^{* n} \rightarrow 0$ strongly while the second summand converges to $C h+\Theta_{T} \sum_{k=0}^{\infty} a_{k} z^{k}$.

We remark that there is a version of the theory of characteristic functions for oneparameter semigroups of contractions which is closely related to Lax-Phillips scattering theory (cf. [LP], Chapter III). We do not discuss it here because our only intention in this section has been to provide an analogue for the following developments and for this it is enough to consider the most elementary situation.

2. Decompositions of Beurling type. Let $\left(\alpha_{t}\right)_{t \geq 0}$ be an $E_{0}$-semigroup, i.e., a pointwise weak*-continuous semigroup of normal unital $*$-endomorphisms of $\mathcal{B}(\tilde{\mathcal{H}})$, where $\tilde{\mathcal{H}}$ is a complex separable Hilbert space.

Definition 2.1. A decomposition $\tilde{\mathcal{H}}=\mathcal{H} \otimes \mathcal{K}$ is of Beurling type with respect to $\left(\alpha_{t}\right)_{t \geq 0}$ if $\mathbb{I} \otimes \mathcal{B}(\mathcal{K})$ is invariant for $\left(\alpha_{t}\right)$ and if the $E_{0}$-semigroup $\left(\gamma_{t}\right)_{t \geq 0}$ on $\mathcal{B}(\mathcal{K})$, given by

$$
\mathbb{I} \otimes \gamma_{t}(y)=\alpha_{t}(\mathbb{I} \otimes y) \quad \text { for all } t \geq 0, y \in \mathcal{B}(\mathcal{K}),
$$

is conjugate to $\left(\alpha_{t}\right)$.

As a motivation for this terminology the reader should compare such decompositions with those in Section 1 where the minimal isometric dilation of a $*$-stable contraction is shown to yield an analogous structure directly related to Beurling's theorem. Here we have a tensor product instead of a direct sum and we consider mappings of a higher level. Nevertheless, the analogy is useful as we shall see.

To construct and classify decompositions of Beurling type it is necessary to make their structure more explicit. Conjugacy of $\left(\alpha_{t}\right)$ and $\left(\gamma_{t}\right)$ means that there is a unitary $w: \mathcal{H} \otimes \mathcal{K} \rightarrow \mathcal{K}$ such that

$$
\alpha_{t}(x)=w^{*} \gamma_{t}\left(w x w^{*}\right) w \quad \text { for all } x \in \mathcal{B}(\mathcal{H} \otimes \mathcal{K}) .
$$

Let $\delta \in \mathcal{H}$ be a unit vector. Then we have an embedding $\mathcal{K} \simeq \delta \otimes \mathcal{K} \subset \mathcal{H} \otimes \mathcal{K}$. The orthogonal projection from $\tilde{\mathcal{H}}=\mathcal{H} \otimes \mathcal{K}$ onto $\mathcal{K}$ is denoted by $q$. By $\hat{w} \in \mathcal{B}(\mathcal{H} \otimes \mathcal{K})$ we denote the isometry onto $\mathcal{K}$ given by $w$, i.e., $\hat{w} \xi=\delta \otimes w \xi$ for all $\xi \in \mathcal{H} \otimes \mathcal{K}$. Then with $I d=I d_{\mathcal{H}}$ we get

$$
\alpha_{t}(x)=\hat{w}^{*}\left(I d \otimes \gamma_{t}\right)\left(\hat{w} x \hat{w}^{*}\right) \hat{w}=u_{t}\left(I d \otimes \gamma_{t}\right)(x) u_{t}^{*},
$$

where $u_{t}=\hat{w}^{*}\left(I d \otimes \gamma_{t}\right)(\hat{w})$. The $E_{0}$-semigroup $\left(I d \otimes \gamma_{t}\right)_{t \geq 0}$ is called an amplification of $\left(\gamma_{t}\right)_{t \geq 0}$, and it is easily checked that $\left(u_{t}\right)_{t \geq 0}$ is a left unitary $\left(I d \otimes \gamma_{t}\right)$-cocycle, i.e., a strongly continuous family $\left(u_{t}\right)_{t \geq 0}$ of unitaries on $\mathcal{H} \otimes \mathcal{K}$ satisfying

$$
u_{t+s}=u_{t}\left(I d \otimes \gamma_{t}\right)\left(u_{s}\right) \quad \text { for all } t, s \geq 0, u_{0}=\mathbb{I} .
$$

In fact, for every isometry $\hat{w} \in \mathcal{B}(\mathcal{H} \otimes \mathcal{K})$ with $\hat{w} \hat{w}^{*}=q$ the formula $u_{t}=\hat{w}^{*}\left(I d \otimes \gamma_{t}\right)(\hat{w})$ always defines such a cocycle. This expresses the fact that an amplification $\left(I d \otimes \gamma_{t}\right)$ is always cocycle conjugate to $\left(\gamma_{t}\right)$. See [Ar], 2.2.4 and 2.2.5, for more details. In this paper, by 'cocycle' we always mean a strongly continuous left unitary cocycle, as above.

The second ingredient of a decomposition of Beurling type is the fact that $\left(\gamma_{t}\right)$ is obtained from $\left(\alpha_{t}\right)$ by restriction. We can write this property in terms of the cocycle. In 
fact, for all $t \geq 0, y \in \mathcal{B}(\mathcal{K})$ we have

$$
\alpha_{t}(\mathbb{I} \otimes y)=u_{t}\left(I d \otimes \gamma_{t}\right)(\mathbb{I} \otimes y) u_{t}^{*}=u_{t}\left(\mathbb{I} \otimes \gamma_{t}(y)\right) u_{t}^{*}
$$

and this always equals $\mathbb{I} \otimes \gamma_{t}(y)$ if and only if $u_{t}$ commutes with $\mathbb{I} \otimes \gamma_{t}(\mathcal{B}(\mathcal{K})$ ) (for all $t \geq 0$ ). The following definition helps to summarize these observations.

Definition 2.2. A $\left(I d \otimes \gamma_{t}\right)$-cocycle $\left(u_{t}\right)_{t \geq 0}$ is called adapted if $u_{t}$ commutes with $\mathbb{I} \otimes$ $\gamma_{t}(\mathcal{B}(\mathcal{K})$ ) (for all $t \geq 0$ ). It is called exact with respect to $\mathcal{K}$ if there is an isometry $\hat{w} \in \mathcal{B}(\mathcal{H} \otimes \mathcal{K})$ onto $\mathcal{K}$ such that

$$
u_{t}=\hat{w}^{*}\left(I d \otimes \gamma_{t}\right)(\hat{w}) \quad \text { for all } t \geq 0 .
$$

Our discussion above yields the following result.

TheOrem 2.3. The decomposition $\mathcal{H} \otimes \mathcal{K}$ is of Beurling type with respect to $\left(\alpha_{t}\right)$, such that $\mathbb{I} \otimes \gamma_{t}(y)=\alpha_{t}(\mathbb{I} \otimes y)$ for all $t \geq 0, y \in \mathcal{B}(\mathcal{K})$, if and only if there exists a $\left(I d \otimes \gamma_{t}\right)$-cocycle $\left(u_{t}\right)$ which is adapted and exact with respect to $\mathcal{K}$, such that $\alpha_{t}(\cdot)=u_{t}\left(\operatorname{Id} \otimes \gamma_{t}\right)(\cdot) u_{t}^{*}$.

We can reinterpret Theorem 2.3 in the following way. To find all decompositions of Beurling type for an $E_{0}$-semigroup, start with (its conjugate version) $\left(\gamma_{t}\right)$ on $\mathcal{B}(\mathcal{K})$ and look for all adapted and exact cocycles of its amplifications. Thus the emphasis of our study shifts to cocycles.

Let us add some comments on adaptedness and exactness according to Definition 2.2. Recall that a $\left(\beta_{t}\right)$-cocycle $\left(u_{t}\right)_{t \geq 0}$ for an $E_{0}$-semigroup $\left(\beta_{t}\right)_{t \geq 0}$ is local in the sense of Powers $[\mathrm{Po}]$ or a gauge cocycle as in $[\mathrm{Ar}], 2.8$, if $u_{t}$ commutes with the range of $\beta_{t}$ (for all $t \geq 0)$. If in our setting $\mathcal{H}=\mathbb{C}$ then we can identify $\left(I d \otimes \gamma_{t}\right)$ and $\left(\gamma_{t}\right)$, and in this case adapted cocycles are nothing but gauge cocycles. But for $\operatorname{dim} \mathcal{H} \geq 2$ we have a strict inclusion of $\mathbb{I} \otimes \gamma_{t}(\mathcal{B}(\mathcal{K}))$ into $\left(I d \otimes \gamma_{t}\right)(\mathcal{B}(\tilde{\mathcal{H}}))$ and thus an adapted cocycle may fail to be a gauge cocycle. The term 'adapted' comes from stochastic processes, i.e., from the possibility to construct adapted quantum stochastic processes by using such cocycles. See our introduction and also [Bh], Chapter 9.

In a similar sense our notion of exactness with respect to $\mathcal{K}$ is a slight generalization of the usual notion of exactness which is obtained for $\mathcal{H}=\mathbb{C}$, i.e., $q=\mathbb{I}$ (cf. [Ar], 2.2.2). Exactness does not depend on the choice of the unit vector $\delta \in \mathcal{H}$. This can be seen by the following reformulation of the definition which is clearly equivalent to the original one and does not involve $\delta$. If $w: \mathcal{H} \otimes \mathcal{K} \rightarrow \mathcal{K}$ is a unitary then define $\left(I d \otimes \gamma_{t}\right)(w): \mathcal{H} \otimes \mathcal{K} \rightarrow \mathcal{K}$ to be the operator which maps $\xi \otimes \eta$ to $\gamma_{t}\left(w_{\xi}\right) \eta$, where $w_{\xi}: \mathcal{K} \rightarrow \mathcal{K}, \eta \mapsto w(\xi \otimes \eta)$. With this notation we can say that $\left(u_{t}\right)$ is exact with respect to $\mathcal{K}$ if there is a unitary $w: \mathcal{H} \otimes \mathcal{K} \rightarrow \mathcal{K}$ such that $u_{t}=w^{*}\left(I d \otimes \gamma_{t}\right)(w)$ for all $t \geq 0$.

3. Convergent cocycles. In this short section we discuss a simple analytic property which is sufficient for a $\left(I d \otimes \gamma_{t}\right)$-cocycle $\left(u_{t}\right)$ to be exact with respect to $\mathcal{K}$. We continue to use the notation of the previous section.

Definition 3.1. A $\left(\beta_{t}\right)$-cocycle $\left(u_{t}\right)$ for an $E_{0}$-semigroup $\left(\beta_{t}\right)$ is called convergent if $u_{t}^{*}$ converges for $t \rightarrow \infty$ in the strong operator topology.

Proposition 3.2. If a $\left(I d \otimes \gamma_{t}\right)$-cocycle $\left(u_{t}\right)$ is convergent and $u_{t}^{*} \rightarrow \hat{w}$ with $\hat{w} \hat{w}^{*}=q$, then $\left(u_{t}\right)$ is exact with respect to $\mathcal{K}$. 
Proof. From the cocycle equation

$$
u_{t}=u_{t+s}\left(I d \otimes \gamma_{t}\right)\left(u_{s}^{*}\right),
$$

which for $s \rightarrow \infty$ converges weakly to $\hat{w}^{*}\left(I d \otimes \gamma_{t}\right)(\hat{w})$, we conclude that $u_{t}=$ $\hat{w}^{*}\left(I d \otimes \gamma_{t}\right)(\hat{w})$ for all $t \geq 0$, i.e., $\left(u_{t}\right)$ is exact with respect to $\mathcal{K}$.

Note that, for a convergent cocycle, $\left(u_{t}\right)$ always converges in the weak operator topology. Recall that in the unitary group strong and weak convergence coincide. But in our applications typically the limit is not unitary and $\left(u_{t}\right)$ does not converge strongly. In this respect the following observation is useful.

LEMma 3.3. Let $\left(x_{t}\right)_{t \geq 0}$ be unitaries converging to $x$ in the strong operator topology. Then a vector $\xi$ is in the range of $x$ if and only if $\left(x_{t}^{*}(\xi)\right)$ is convergent in norm.

The lemma can be proved by combining the following elementary facts: $\left(x_{t}^{*}\right)$ converges to $x^{*}$ in the weak operator topology. Weakly convergent unit vectors are convergent in norm if and only if the limit is a unit vector. $\xi$ is in the range of $x$ if and only if $\left\|x^{*}(\xi)\right\|=\|\xi\|$.

4. CCR-flows. The most basic examples of (non-automorphic) $E_{0}$-semigroups are the CCR-flows and they can be realized on symmetric Fock spaces. See [Pa] for details on Fock space and $[\mathrm{Ar}]$ for a presentation of CCR-flows from the point of view of $E_{0}$-semigroups. We want to discuss decompositions of Beurling type in this case.

Let us introduce some notation. We denote by $\mathcal{F}:=\Gamma\left(L^{2}[0, \infty), k\right)$ the symmetric Fock space over $L^{2}[0, \infty)$ with a multiplicity space $k$. For all $t \geq 0$ we have a canonical decomposition $\mathcal{F}=\mathcal{F}_{t]} \otimes \mathcal{F}_{[t}$, where $\mathcal{F}_{t]}:=\Gamma\left(L^{2}[0, t), k\right)$ and $\mathcal{F}_{[t}:=\Gamma\left(L^{2}[t, \infty), k\right)$. There is also a canonical way to identify $\mathcal{F}$ and $\mathcal{F}_{[t}$ using the right shift. Always suppressing the notation for the identification maps we can define

$$
\gamma_{t}: \mathcal{B}(\mathcal{F}) \rightarrow \mathcal{B}(\mathcal{F}), \quad x \mapsto \mathbb{I}_{t]} \otimes x,
$$

where $\mathbb{I}_{t]}$ is the identity on $\mathcal{F}_{t]}$. The $E_{0}$-semigroup $\left(\gamma_{t}\right)_{t \geq 0}$ is called the CCR-flow of index $\operatorname{dim} k$. In $[\mathrm{Po}]$, Powers gave the following abstract characterization: An $E_{0}$-semigroup which is completely spatial and in standard form is conjugate to a CCR-flow. See [Ar], 2.6 and 2.7, for complete spatiality. 'Standard form' means that there exists an absorbing vector state. For a CCR-flow it is given by the vacuum vector $\Omega \in \mathcal{F}$, and the absorbing property means that

$$
\rho \circ \gamma_{t} \rightarrow\langle\Omega, \Omega\rangle \quad(t \rightarrow \infty)
$$

for all normal states $\rho$. Note that an absorbing state is always a unique invariant state.

Now let $\mathcal{H}$ be another Hilbert space and assume that the $E_{0}$-semigroup $\left(\alpha_{t}\right)_{t \geq 0}$ on $\mathcal{B}(\mathcal{H} \otimes \mathcal{F})$ provides a decomposition of Beurling type such that $\mathbb{I} \otimes \gamma_{t}(y)=\alpha_{t}(\mathbb{I} \otimes y)$ for all $t \geq 0, y \in \mathcal{B}(\mathcal{F})$. In particular, $\left(\alpha_{t}\right)$ and $\left(\gamma_{t}\right)$ are conjugate and there is also an absorbing vector state given by $\tilde{\Omega} \in \mathcal{H} \otimes \mathcal{F}$ for $\left(\alpha_{t}\right)$.

LEMMA 4.1. There is a unit vector $\delta \in \mathcal{H}$ such that $\tilde{\Omega}=\delta \otimes \Omega$.

Proof. For all $y \in \mathcal{B}(\mathcal{F})$ we obtain

$$
\langle\tilde{\Omega},(\mathbb{I} \otimes y) \tilde{\Omega}\rangle=\left\langle\tilde{\Omega}, \alpha_{t}(\mathbb{I} \otimes y) \tilde{\Omega}\right\rangle=\left\langle\tilde{\Omega},\left(\mathbb{I} \otimes \gamma_{t}\right)(y) \tilde{\Omega}\right\rangle \stackrel{t \rightarrow \infty}{\longrightarrow}\langle\Omega, y \Omega\rangle .
$$


We conclude that $\langle\tilde{\Omega},(\mathbb{I} \otimes y) \tilde{\Omega}\rangle=\langle\Omega, y \Omega\rangle$ for all $y \in \mathcal{B}(\mathcal{F})$. This means that $\langle\tilde{\Omega}, \cdot \tilde{\Omega}\rangle$ is not an entangled state. In other words, there is a unit vector $\delta \in \mathcal{H}$ such that $\tilde{\Omega}=\delta \otimes \Omega$.

From Lemma 4.1 we see that here we have a canonical choice for the unit vector $\delta \in \mathcal{H}$ which was chosen arbitrarily in Section 2. This particular choice will be used in the following, together with the canonical embeddings

$$
\begin{aligned}
\mathcal{H} & \simeq \mathcal{H} \otimes \Omega \subset \mathcal{H} \otimes \mathcal{F}=\tilde{\mathcal{H}}, \\
\mathcal{F} & \simeq \delta \otimes \mathcal{F} \subset \mathcal{H} \otimes \mathcal{F}=\tilde{\mathcal{H}} .
\end{aligned}
$$

We denote by $p=\mathbb{I}_{\mathcal{H}} \otimes|\Omega\rangle\langle\Omega|$ the orthogonal projection with range $\mathcal{H}$ and by $q=$ $|\delta\rangle\langle\delta| \otimes \mathbb{I}_{\mathcal{F}}$ the orthogonal projection with range $\mathcal{F}$.

Now we can give some equivalent characterizations of decompositions of Beurling type for CCR-flows. Note that by Theorem 2.3 the presentation $\alpha_{t}(\cdot)=u_{t}\left(I d \otimes \gamma_{t}\right)(\cdot) u_{t}^{*}$ with an adapted cocycle $\left(u_{t}\right)$ is not a restriction of generality. Let us call the cocycle $\left(\hat{u}_{t}\right)$ a modification of $\left(u_{t}\right)$ if there is a gauge cocycle $\left(\hat{v}_{t}\right)$ such that $\hat{u}_{t}=u_{t} \hat{v}_{t}$ for all $t \geq 0$. Then also $\alpha_{t}(\cdot)=\hat{u}_{t}\left(I d \otimes \gamma_{t}\right)(\cdot) \hat{u}_{t}^{*}$. Further we need the compression of $\alpha_{t}$ to $\mathcal{H}$, i.e.,

$$
Z_{t}: \mathcal{B}(\mathcal{H}) \rightarrow \mathcal{B}(\mathcal{H}), \quad x \mapsto p \alpha_{t}(p x p) p=p \alpha_{t}(x p) p
$$

which is a unital completely positive map (for each $t \geq 0$ ).

THEOREM 4.2. Let $\left(\gamma_{t}\right)$ be the CCR-flow on $\mathcal{F}$ and $\left(\alpha_{t}\right)$ another $E_{0}$-semigroup on $\mathcal{H} \otimes \mathcal{F}$ given by $\alpha_{t}(\cdot)=u_{t}\left(I d \otimes \gamma_{t}\right)(\cdot) u_{t}^{*}$ with an adapted $\left(I d \otimes \gamma_{t}\right)$-cocycle $\left(u_{t}\right)$. The following assertions are equivalent:

(a) $\tilde{\mathcal{H}}=\mathcal{H} \otimes \mathcal{F}$ is a decomposition of Beurling type with respect to $\left(\alpha_{t}\right)$, such that $\mathbb{I} \otimes \gamma_{t}(y)=\alpha_{t}(\mathbb{I} \otimes y)$ for all $t \geq 0, y \in \mathcal{B}(\mathcal{F})$.

(b) There exists a modification $\left(\hat{u}_{t}\right)$ of $\left(u_{t}\right)$ which is exact with respect to $\mathcal{F}$, i.e., $\hat{u}_{t}=$ $\hat{w}^{*}\left(I d \otimes \gamma_{t}\right)(\hat{w})$, where $\hat{w} \hat{w}^{*}=q$.

(c) There exists a modification $\left(\hat{u}_{t}\right)$ of $\left(u_{t}\right)$ which is convergent, such that $\hat{u}_{t}^{*} \rightarrow \hat{w}$, where $\hat{w} \hat{w}^{*}=q$.

(d) $\left(Z_{t}\right)_{t \geq 0}$ is a unital CP-semigroup with an absorbing vector state (given by $\delta \in \mathcal{H}$ ).

(e) $\left(Z_{t}\right)_{t \geq 0}$ is an ergodic unital CP-semigroup with an invariant vector state (given by $\delta \in \mathcal{H})$. 'Ergodic' means that the only fixed points for all $Z_{t}$ are scalar multiples of $\mathbb{I}_{\mathcal{H}}$.

We see that under the conditions of Theorem 4.2 we have a converse of Proposition 3.2. Moreover, the analogy to the Hilbert space theory in Section 1 becomes more explicit. We can think of $\left(\alpha_{t}\right)$ as a homomorphic dilation of a semigroup $\left(Z_{t}\right)$ of unital CP-maps and this semigroup already determines whether we have a decomposition of Beurling type. Moreover we have [in part (c)] a limit formula which is parallel to the one discussed in Section 1 and we may think of $\hat{w} \in \mathcal{B}(\mathcal{H} \otimes \mathcal{F})$ or the corresponding $w: \mathcal{H} \otimes \mathcal{F} \rightarrow \mathcal{F}$ as an analogue of the characteristic function.

Proof.

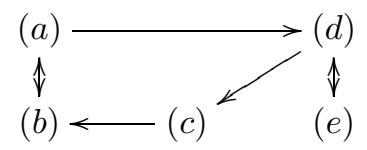


The equivalence $(\mathrm{a}) \Leftrightarrow(\mathrm{b})$ is a reformulation of Theorem 2.3 which we have repeated here for convenience. $(\mathrm{c}) \Rightarrow(\mathrm{b})$ is Proposition 3.2.

Let us now show $(\mathrm{a}) \Rightarrow(\mathrm{d})$. The semigroup property of $\left(Z_{t}\right)$ follows from

$$
Z_{t} Z_{s}(x)=p \alpha_{t}\left(p \alpha_{s}(x) p\right) p=p \alpha_{t}(p) \alpha_{t+s}(x) \alpha_{t}(p) p
$$

together with

$$
\begin{gathered}
\alpha_{t}(p)=u_{t}\left(I d \otimes \gamma_{t}\right)(p) u_{t}^{*}=u_{t}\left(I d \otimes \gamma_{t}\right)\left(\mathbb{I}_{\mathcal{H}} \otimes|\Omega\rangle\langle\Omega|\right) u_{t}^{*} \\
=u_{t}\left(\mathbb{I}_{\mathcal{H}} \otimes \gamma_{t}(|\Omega\rangle\langle\Omega|)\right) u_{t}^{*}=\mathbb{I}_{\mathcal{H}} \otimes \gamma_{t}(|\Omega\rangle\langle\Omega|)=\mathbb{I}_{\mathcal{H}} \otimes \mathbb{I}_{t]} \otimes|\Omega\rangle\langle\Omega| \geq p,
\end{gathered}
$$

where we used the adaptedness of the cocycle $\left(u_{t}\right)$. By Lemma 4.1 we know that $\delta \otimes \Omega$ yields an absorbing vector state for $\left(\alpha_{t}\right)$. Thus for any normal state $\rho$ on $\mathcal{B}(\mathcal{H})$

$$
\rho \circ Z_{t}(x)=(\rho \otimes\langle\Omega, \cdot \Omega\rangle) \circ \alpha_{t}(x p) \stackrel{t \rightarrow \infty}{\longrightarrow}\langle\delta \otimes \Omega,(x p) \delta \otimes \Omega\rangle=\langle\delta, x \delta\rangle,
$$

i.e., $\langle\delta, \cdot \delta\rangle$ is an absorbing vector state for $\left(Z_{t}\right)$.

$(\mathrm{d}) \Leftrightarrow(\mathrm{e})$ is well known, see for example [Go], A.5.2. For convenience we sketch briefly the main arguments. For a nontrivial fixed point we can find two normal states assigning different values to it which clearly rules out the absorption property. Conversely, start with the observation that for an invariant state $\langle\delta, \cdot \delta\rangle$ we always have $|\delta\rangle\langle\delta| \leq Z_{t}(|\delta\rangle\langle\delta|)$ and thus there is a strong limit of $\left(Z_{t}(|\delta\rangle\langle\delta|)\right)$ for $t \rightarrow \infty$. Because this limit is a fixed point, ergodicity implies that it coincides with $\mathbb{I}_{\mathcal{H}}$. From this it follows that the invariant state is absorbing.

It remains to prove $(\mathrm{d}) \Rightarrow(\mathrm{c})$. We start by showing that there is a modification $\left(\hat{u}_{t}\right)$ of $\left(u_{t}\right)$ such that

$$
\hat{u}_{t} \delta \otimes \Omega=\delta \otimes \Omega \quad \text { for all } t \geq 0 .
$$

We have for all $t \geq 0$ and $x \in \mathcal{B}(\mathcal{H})$

$$
Z_{t}(x)=p u_{t}\left(I d \otimes \gamma_{t}\right)(x p) u_{t}^{*} p=p u_{t}(x \otimes \mathbb{I}) u_{t}^{*} p
$$

[using the definition of $\left(\gamma_{t}\right)$ and adaptedness of $\left.\left(u_{t}\right)\right]$ and thus

$$
\left\langle u_{t}^{*} \delta \otimes \Omega,(x \otimes \mathbb{I}) u_{t}^{*} \delta \otimes \Omega\right\rangle=\left\langle\delta \otimes \Omega, u_{t}(x \otimes \mathbb{I}) u_{t}^{*} \delta \otimes \Omega\right\rangle=\left\langle\delta, Z_{t}(x) \delta\right\rangle=\langle\delta, x \delta\rangle .
$$

We conclude that for all $t \geq 0$ we have

$$
u_{t}^{*} \delta \otimes \Omega=\delta \otimes \hat{\Omega}_{t},
$$

where $\hat{\Omega}_{t} \in \mathcal{F}_{t]}$ is a unit vector. The cocycle property of $\left(u_{t}\right)$ implies that

$$
\hat{\Omega}_{t} \otimes \hat{\Omega}_{s}=\hat{\Omega}_{t+s}
$$

(using the canonical identifications with respect to $\mathcal{F}_{t]} \otimes \mathcal{F}_{s]}=\mathcal{F}_{t+s]}$ ). This means that we can think of $\left(\hat{\Omega}_{t}\right)_{t \geq 0}$ as a unit. Explicitly, in Arveson's sense (cf. [Ar], 2.5) the semigroup $\left(s_{t}\right)_{t \geq 0}$ given by the isometries

$$
s_{t}: \mathcal{F} \rightarrow \mathcal{F}, \quad \eta \mapsto \hat{\Omega}_{t} \otimes \eta
$$

is a unit for $\left(\gamma_{t}\right)$. It is known that the gauge cocycles act transitively on this kind of units, see $[\mathrm{Ar}], 3.8 .5$. In particular, we can find a gauge cocycle $\left(v_{t}\right)_{t \geq 0}$ of $\left(\gamma_{t}\right)$ which maps the 
distinguished unit $\left(\Omega_{t}\right)_{t \geq 0}$ obtained by factorization of the vacuum vector $\Omega$ to the unit $\left(\hat{\Omega}_{t}\right)_{t \geq 0}$. Now we define the modification $\left(\hat{u}_{t}\right)$ of $\left(u_{t}\right)$ by

$$
\hat{u}_{t}=u_{t}\left(\mathbb{I} \otimes v_{t}\right) \quad \text { for all } t \geq 0 .
$$

Then $\hat{u}_{t} \delta \otimes \Omega=\delta \otimes \Omega$, as claimed.

Now because $\langle\delta, \cdot \delta\rangle$ is absorbing for $\left(Z_{t}\right)$, we can start with an arbitrary unit vector $\xi \in \mathcal{H}$, and for all $x \in \mathcal{B}(\mathcal{H})$ we get

$$
\left\langle\hat{u}_{t}^{*} \xi \otimes \Omega,(x \otimes \mathbb{I}) \hat{u}_{t}^{*} \xi \otimes \Omega\right\rangle=\left\langle\xi, Z_{t}(x) \xi\right\rangle \stackrel{t \rightarrow \infty}{\longrightarrow}\langle\delta, x \delta\rangle .
$$

If we choose for $x$ the one-dimensional projection $|\delta\rangle\langle\delta|$ then it follows that

$$
\left\|(|\delta\rangle\langle\delta| \otimes \mathbb{I}) \hat{u}_{t}^{*} \xi \otimes \Omega\right\|^{2} \stackrel{t \rightarrow \infty}{\longrightarrow}\langle\delta, \delta\rangle\langle\delta, \delta\rangle=1
$$

and because $|\delta\rangle\langle\delta| \otimes \mathbb{I}=q$ we have

$$
\left\|q \hat{u}_{t}^{*} \xi \otimes \Omega\right\| \stackrel{t \rightarrow \infty}{\longrightarrow} 1 .
$$

This means that we can choose vectors $\eta_{t} \in \mathcal{F}_{t]}$ such that

$$
\Delta_{t}:=\left\|\hat{u}_{t}^{*} \xi \otimes \Omega-\delta \otimes \eta_{t}\right\| \stackrel{t \rightarrow \infty}{\longrightarrow} 0 .
$$

Then for $t, s \geq 0$

$$
\begin{gathered}
\left\|\left(\hat{u}_{t+s}^{*}-\hat{u}_{t}^{*}\right) \xi \otimes \Omega\right\|=\left\|\left[\left(I d \otimes \gamma_{t}\right)\left(\hat{u}_{s}^{*}\right) \hat{u}_{t}^{*}-\hat{u}_{t}^{*}\right] \xi \otimes \Omega\right\| \\
\leq 2 \Delta_{t}+\left\|\left(I d \otimes \gamma_{t}\right)\left(\hat{u}_{s}^{*}\right) \delta \otimes \eta_{t}-\delta \otimes \eta_{t}\right\|=2 \Delta_{t} .
\end{gathered}
$$

Indeed, $\left(I d \otimes \gamma_{t}\right)\left(\hat{u}_{s}^{*}\right) \delta \otimes \eta_{t}=\delta \otimes \eta_{t}$ because $\eta_{t} \in \mathcal{F}_{t]}$ and $\hat{u}_{s}^{*} \delta \otimes \Omega=\delta \otimes \Omega$. We conclude that $\left(\hat{u}_{t}^{*} \xi \otimes \Omega\right)_{t \geq 0}$ is a Cauchy net and thus convergent.

We have to prove that $\left(\hat{u}_{t}^{*} \hat{\xi}\right)$ is convergent for all $\hat{\xi} \in \mathcal{H} \otimes \mathcal{F}$. But this can be reduced to the special case above as follows. First, it is enough to consider $\hat{\xi}=\xi^{\prime} \otimes \eta_{t}^{\prime}$ with $\eta_{t}^{\prime} \in \mathcal{F}_{t]}$ for all $t$, because linear combinations of such vectors are dense in $\mathcal{H} \otimes \mathcal{F}$. Second, we have

$$
\hat{u}_{t+s}^{*}\left(\xi^{\prime} \otimes \eta_{t}^{\prime}\right)=\left(I d \otimes \gamma_{t}\right)\left(\hat{u}_{s}^{*}\right) \hat{u}_{t}^{*}\left(\xi^{\prime} \otimes \eta_{t}^{\prime}\right)
$$

and because $\hat{u}_{t}^{*}\left(\xi^{\prime} \otimes \eta_{t}^{\prime}\right)$ can be approximated by linear combinations of vectors of the form $\xi \otimes \eta_{t}$ with $\eta_{t} \in \mathcal{F}_{t]}$ we see that convergence of $\hat{u}_{t+s}^{*}\left(\xi^{\prime} \otimes \eta_{t}^{\prime}\right)$ for $s \rightarrow \infty$ always takes place if $\hat{u}_{s}^{*}(\xi \otimes \Omega)$ converges for all $\xi \in \mathcal{H}$. But the latter has already been shown.

We have established the strong convergence of $\left(\hat{u}_{t}^{*}\right)$, i.e., convergence of the cocycle in the sense of Definition 3.1. As a strong limit of unitaries, $\hat{w}:=\lim _{n \rightarrow \infty} \hat{u}_{t}^{*}$ is an isometry. Further we have for all unit vectors $\hat{\xi} \in \mathcal{H} \otimes \mathcal{F}$

$$
\left\|q \hat{u}_{t}^{*} \hat{\xi}\right\| \stackrel{t \rightarrow \infty}{\longrightarrow} 1 .
$$

This has been shown for $\hat{\xi}=\xi \otimes \Omega$ before and in general it follows by the approximations above. It implies that the range of $\hat{w}$ is contained in $\mathcal{F}$.

Finally, we have to show the opposite inclusion, i.e., $\mathcal{F}$ is contained in the range of $\hat{w}$. For this, note that linear combinations of vectors of the form $\delta \otimes \eta_{t}$ with $\eta_{t} \in \mathcal{F}_{t]}$ for all $t$, are dense in $\delta \otimes \mathcal{F} \simeq \mathcal{F}$. From $\hat{u}_{s}(\delta \otimes \Omega)=\delta \otimes \Omega$ we get

$$
\hat{u}_{t+s}\left(\delta \otimes \eta_{t}\right)=\hat{u}_{t}\left(I d \otimes \gamma_{t}\right)\left(\hat{u}_{s}\right)\left(\delta \otimes \eta_{t}\right)=\hat{u}_{t}\left(\delta \otimes \eta_{t}\right)
$$

We infer that for all $\eta \in \mathcal{F}$ also $\left(\hat{u}_{t} \eta\right)$ is convergent. Thus our proof can be finished by applying Lemma 3.3 . 


\section{References}

[Ar] W. Arveson, Noncommutative Dynamics and E-Semigroups, Springer Monographs in Mathematics, 2003.

[Bh] B. V. R. Bhat, Cocycles of CCR flows, Mem. Am. Math. Soc. 709 (2001).

[FF] C. Foiaş and A. E. Frazho, The Commutant Lifting Approach to Interpolation Problems, Birkhäuser, 1990.

[Go] R. Gohm, Noncommutative Stationary Processes, Lecture Notes in Mathematics 1839, Springer-Verlag, 2004.

[Li] J. M. Lindsay, Quantum stochastic analysis - an introduction, in: Quantum Independent Increment Processes I, U. Franz and M. Schürmann (eds.), Lecture Notes in Mathematics 1865, Springer, 2005.

[LP] P. D. Lax and R. S. Phillips, Scattering Theory, Academic Press, 1967.

[Ni] N. K. Nikolskij, Treatise on the Shift Operator. Spectral Function Theory, Springer-Verlag, 1986.

[Pa] K. R. Parthasarathy, An Introduction to Quantum Stochastic Calculus, Birkhäuser, 1992.

[Po] R. T. Powers, New examples of continuous spatial semigroups of $*$-endomorphisms of $\mathcal{B}(\mathcal{H})$, Int. J. Math. 10 (1999), 215-288. 